\title{
Neuronal Stability and Drift across Periods of Sleep: Premotor Activity Patterns in a Vocal Control Nucleus of Adult Zebra Finches
}

\author{
Peter L. Rauske, ${ }^{1}$ Zhiyi Chi, ${ }^{2}$ Amish S. Dave, ${ }^{1}$ and Daniel Margoliash ${ }^{1}$ \\ Departments of ${ }^{1}$ Organismal Biology and Anatomy and ${ }^{2}$ Statistics, University of Chicago, Chicago, Illinois 60611
}

\begin{abstract}
How stable are neural activity patterns compared across periods of sleep? We evaluated this question in adult zebra finches, whose premotor neurons in the nucleus robustus arcopallialis (RA) exhibit sequences of bursts during daytime singing that are characterized by precise timing relative to song syllables. Each burst has a highly regulated pattern of spikes. We assessed these spike patterns in singing that occurred before and after periods of sleep. For about half of the neurons, one or more premotor bursts had changed after sleep, an average of $20 \%$ of all bursts across all RA neurons. After sleep, modified bursts were characterized by a discrete, albeit modest, loss of spikes with compensatory increases in spike intervals, but not changes in timing relative to the syllable. Changes in burst structure followed both interrupted bouts of sleep (1.5-3 h) and full nights of sleep, implicating sleep and not circadian cycle as mediating these effects. Changes in burst structure were also observed during the day, but far less frequently. In cases where multiple bursts in the sequence changed in a single cell, the sequence position of those bursts tended to cluster together. Bursts that did not show discrete changes in structure also showed changes in spike counts, but not biased toward losses. We hypothesize that changes in burst patterns during sleep represent active sculpting of the RA network, supporting auditory feedback-mediated song maintenance.
\end{abstract}

\section{Introduction}

Sleep-dependent behavioral plasticity has been observed in a broad range of perceptual, motor, and higher-level cognitive tasks in studies in adult humans (Karni et al., 1994; Stickgold et al., 2000; Fischer et al., 2002; Walker et al., 2002; Fenn et al., 2003; Wagner et al., 2004; Brawn et al., 2008). Electrophysiological studies support a role for active processes during sleep affecting memory consolidation in humans (Maquet et al., 2000; Peigneux et al., 2004; Reis et al., 2009), and behavioral and electrophysiological studies in animals implicate sleep in plastic mechanisms. Sleep modulates plastic changes in ocular dominance histograms in the developing visual cortex of young cats (Frank et al., 2001; Aton et al., 2009), the emergence of song system neuronal bursting in juvenile birds at the onset of song learning (Shank and Margoliash, 2009), and experience-dependent changes in the correlations of activity patterns of rat hippocampal neurons (Poe et al., 2000).

These results emphasize changes measured in populations of neurons. Sleep-dependent changes in the individual activity patterns of single neurons during behavior are not well defined, however, and thus there is little data on the stability of single neuron activity patterns across periods of sleep. In this study, we address this issue in the birdsong system. Male zebra finches

Received June 5, 2009; revised Dec. 18, 2009; accepted Jan. 11, 2010.

This work was supported in part by National Institutes of Health Grant MH59831 to D.M. We are grateful to Ana Amador, Henry D. I. Abarbanel, and Stephen D. Shea for critical reviews of this manuscript.

Correspondence should be addressed to Dr. Peter L. Rauske, Sensory Motor Performance Program, Rehabilitation Institute of Chicago, 345 E. Superior Street, Suite 1406, Chicago, IL 60611. E-mail: plrauske@gmail.com.

DOI:10.1523/JNEUROSCI.3112-09.2010

Copyright $\odot 2010$ the authors $\quad 0270-6474 / 10 / 302783-12 \$ 15.00 / 0$ court females with "directed" singing: precisely structured, regular songs comprising introductory notes followed by a sequence of syllables organized into a "motif." Directed songs are even more highly regulated than the undirected songs males otherwise sing (Sossinka and Böhner, 1980; Kao et al., 2005; Glaze and Troyer, 2006).

Associated with directed singing are highly structured bursts of activity in presumptive projection neurons in the nucleus robustus arcopallialis (RA) (Yu and Margoliash, 1996). Each spike burst has submillisecond precision in its timing relative to its corresponding syllable within the motif (Chi and Margoliash, 2001; Leonardo and Fee, 2005). These bursts have a well-defined number of spikes in a well-defined temporal pattern, both of which vary across bursts emitted at different times in the song. A given burst thus has a specific identity associated with onset time, number of spikes, and pattern of spikes. RA neurons show highly regulated oscillatory spontaneous activity, become completely suppressed about $50 \mathrm{~ms}$ before onset of song, and may achieve instantaneous firing rates of almost $800 \mathrm{~Hz}$ during singing. Thus, the nervous system expresses almost the entire dynamic range available to precisely modulate the activity of single RA neurons during singing.

We took advantage of the reliability and precision of this system to examine neuronal stability over extended periods of time. RA extracellular recordings can be stable with high signal-tonoise ratio (SNR), but the technical challenge of maintaining high-quality recordings over the required durations and behaviors in freely moving animals required by this design limited the size of the dataset. Nevertheless, we were able to directly compare premotor activity of the same single neurons before and after 
periods of sleep. To the best of our knowledge, such comparisons have not been reported in any premotor system.

\section{Materials and Methods}

To examine the effects of sleep on the stability of premotor burst patterns in RA neurons, we recorded neuronal activity in three types of experimental sessions: short-sleep (or interrupted-sleep) sessions, long-sleep (or normal circadian-sleep) sessions, and awake-only sessions. For both types of sessions including sleep, we recorded the activity of the same single RA neurons while birds sang or produced learned calls (see below) both before and after the period of sleep. We developed algorithms to identify changes to burst patterns across periods of sleep, as well as statistical techniques to compare the frequency of such changes with that observed in the absence of sleep.

Electrophysiology and design of the experiments. All animal procedures were approved by an Institutional Animal Care and Use Committee. Adult male zebra finches $(n=13)$ were habituated to either a $16 / 8 \mathrm{~h}$ or $14 / 10 \mathrm{~h}$ light/dark cycle. We found no systematic differences between the two conditions, and combine the data for aggregate statistical analyses. The birds were implanted with microdrives with electrodes targeting RA; the implant design and surgical procedures have been described in detail previously (Dave et al., 1999). Briefly, a recording device carrying four glass-coated Pt-Ir electrodes (impedance, 1.2-2.0 M $\Omega$ at $1 \mathrm{kHz}$ ) was implanted under modified Equithesin anesthesia over RA. During recording sessions starting 2-4 d later, a flexible cable connected the headgear to an overhead commutator to allow the bird free movement within the cage. Differential recordings were used to minimize movement artifacts. Recording sites were obtained by audiovisual monitoring of the recordings while using a drive screw to manually advance the electrodes. Birds were manually restrained during this procedure, then carefully released into the cage while trying to maintain unit isolation.

Recording sessions began at various times during the day, and we recorded only sites with at least one unit that could be well isolated. In all cases, a conspecific female was introduced into an adjacent half-cage to elicit directed singing and calling. [In male zebra finches, contact or so-called "long" calls are learned vocalizations whose production involves RA premotor activity (Zann, 1985; Simpson and Vicario, 1990), and they are treated equivalently with song syllables in this study.]

After collecting high SNR spike data during vocalizations comprising at least 10 song motifs and/or contact calls, or in the normal circadian rhythm depending on experimental design (see below), the cage lights were doused. After the bird was quiescent for several minutes, activity in RA entered a characteristic bursting mode. This distinct state was never observed in an awake bird, and bursting disappeared whenever the bird was disturbed or became active. Spontaneous bursting in RA and its efferent sensorimotor control nucleus (HVC) has come to be used as an assay for sleep. It is reliably associated with the onset of sleep postures and strong, selective auditory responses (Dave et al., 1998; Dave and Margoliash, 2000; Nick and Konishi, 2001; Hahnloser et al., 2002, 2006; Cardin and Schmidt, 2003; Rauske et al., 2003; Shank and Margoliash, 2009) and has been correlated with EEG measures of sleep (Nick and Konishi, 2001; Hahnloser et al., 2006; Shank and Margoliash, 2009). During recording sessions including 1.5-3 h darkness (labeled "short sleep"; $n=10$ neurons, 4 birds), we recorded continuously from the isolated RA single units, enabling us to estimate the amount of time birds actually slept by examining the bursting activity (or lack thereof) during the dark period. We used a quantitative measure of spontaneous RA bursting as a sleep assay, described below. During some recording sessions, we also verified by direct observation (infrared monitoring) that the bird's eyes were closed and respiration slowed when RA activity indicated sleep (Dave et al., 1998).

During the short-sleep recording sessions, we also presented playback of the bird's own song. Recordings of the bird's own song were scaled to $70 \mathrm{~dB}$ root-mean-squared amplitude and presented randomly at $10-30 \mathrm{~s}$ intervals beginning immediately after turning out the lights. After 50250 repetitions of song playback, we recorded 20-60 min of ongoing spiking activity while the bird remained asleep. Thereafter, the lights were then turned back on, rousing the bird, after $1.5-3 \mathrm{~h}$ of sleep. Birds then directed singing toward the adjacent female, and we continued recordings until single-unit isolation was lost. Auditory stimulation enabled us to verify the responsiveness to the bird's own song that RA neurons exhibit exclusively during sleep (Dave et al., 1998). Furthermore, this was a preliminary experiment to test the hypothesis that sleeprelated changes in singing behavior result from drift arising from neural replay during sleep activity without concomitant auditory feedback (Derégnaucourt et al., 2005). We hypothesized that playback would provide structured activity during sleep, possibly preventing sleep-related changes, but failed to see systematic differences between short-sleep (auditory stimulation) and long-sleep (no stimulation) sessions (see Results), a null result with respect to the sleep-drift hypothesis. We do not consider this hypothesis further in this study.

In some additional, exceptional cases ( $n=5$ neurons, 3 birds), we successfully gambled on our ability to maintain stable unit isolation across a full night of sleep ( 8 or $10 \mathrm{~h}$ ), maintaining the normal light/dark cycle. All but one of these cases involved single-unit isolation, with the exception being a site in which a pair of units could be reliably distinguished from background activity but not from each other; this "doubleunit" site was treated similarly to single units in our analysis. No auditory stimuli were presented during sleep for these sites, but ongoing activity was sampled throughout the night to verify the presence of bursting activity in RA that indicated the bird remained asleep. When the next day's light cycle began, recordings continued until unit isolation was lost.

Finally, we augmented this data set with additional recordings during vocalizations in recording sessions that did not include sleep (see below).

Analysis of sleep. Sleep was objectively defined behaviorally (eye closure, body posture), and we also developed a quantitative measure of spontaneous bursting in RA neurons to use as an assay for sleep. We first established a baseline for a neuron's spontaneous spiking activity during periods before and after darkness when the bird was awake and active, but not vocalizing. We used 1-4 min segments of neuronal activity both before and after darkness, dividing the spiking activity into $3 \mathrm{~s}$ segments. For each segment, the distribution of interspike intervals (ISIs) was approximately Gaussian because of the highly regular spiking activity of RA neurons in awake, nonvocalizing birds. We calculated for each segment's ISI distribution the mean (ISI-MEAN) and standard deviation (ISI-SD). The resulting range of values across all awake segments for each single unit provided an estimate of the baseline variability in spiking activity in the awake bird.

To quantify the amount of sleep during darkness, we similarly divided spiking activity into $3 \mathrm{~s}$ segments, calculating the ISI-MEAN and ISI-SD for each segment. Any segment whose ISI-MEAN and ISI-SD both fell within a $95 \%$ confidence interval as determined by the baseline awake distributions was labeled "awake"; all other segments were labeled as "sleep" (for example, see supplemental Fig. 1, available at www.jneurosci.org as supplemental material). Such labeling agreed well with visual inspection of spiking activity, with segments including sleep-typical depressed firing rates and/or bursting reliably labeled as sleep. Video surveillance under infrared illumination verified that the bird was quiescent with closed eyelids in $>95 \%$ of sleep-labeled segments. We had not developed reliable EEG recording techniques and an understanding of sleep staging in zebra finches except toward the end of these studies (Low et al., 2008); nevertheless, our analysis reliably distinguished sleep from waking.

Song syllables, spike bursts, and a definition of burst types. Vocalizations and onset and offset times for each syllable were identified by manual inspection of spectrographs. A syllable was defined as a stereotyped vocal gesture containing no silent interval $>10 \mathrm{~ms}$; in addition to the traditionally defined song syllables that comprise song "motifs" (stereotyped sequence of syllables), we also included introductory notes at the beginning of singing bouts and isolated "long" calls, both of which recruit RA bursting activity, in our definition of "syllable" for this study. Syllable onset times and spike times were merged for each site to create a raster plot of spiking activity associated with each syllable type. For each syllable, we included the spiking activity beginning $50 \mathrm{~ms}$ before syllable onset and ending with the syllable offset.

We used simple thresholding techniques to identify spike times for most, extremely well-isolated single units. For a few sites with more ambiguous isolation, we used the Spikesort program, which uses a Bayesian approach to identify putative spikes with distinct spike-shape models 
(Lewicki, 1994). To confirm single-unit isolation in all cases, we visually inspected overlaid waveforms from all identified spike times to confirm that spike shapes were consistent throughout our recordings, and we used ISI distributions to confirm the hallmarks of single-unit isolation in RA (i.e., an approximately Gaussian distribution of ISIs during behavioral quiescence and a lack of ISIs $<1 \mathrm{~ms}$ ). For the majority of sites ( 28 of 42 single units), we were able to confidently identify $100 \%$ of all spikes after manual inspection. The remaining single-unit sites, as well as the "double-unit" site, included a small number of ambiguous spikes, so we estimate that we achieved $98-99 \%$ correct classification. In these cases, the ambiguities were attributable to either the extreme attenuation of spike amplitude during bursting (Yu and Margoliash, 1996) or sporadic background spiking activity that could not be reliably distinguished from attenuated spikes in the recordings with the lowest SNR. These sites, however, did not show any greater or lesser stability of temporal patterns of spike bursts - the principal dependent variable of this study - than did those sites with completely reliable spike identification.

RA activity during singing is characterized as having high-frequency bursts of spikes organized into trains of bursts. Each burst in the train of bursts is distinguished from the others both by the pattern of spikes and the timing of the burst relative to the syllable (Yu and Margoliash, 1996; Dave and Margoliash, 2000; Leonardo and Fee, 2005). In this study, we defined a burst as a sequence of consecutive spikes with all interspike intervals $<10 \mathrm{~ms}$. This simple definition reliably identified all bursts of two or more spikes of an RA neuron during singing. In all cases, we also could readily identify a canonical sequence of bursts for each syllable (Yu and Margoliash, 1996). Aligning multiple renditions of the sequences of bursts relative to the onset of a given syllable (as in a raster plot) created stacks of bursts, with each "burst stack" associated with a particular time relative to syllable onset and a particular temporal pattern of spikes. We identified $2.1 \pm 1.3$ bursts for each syllable across all the neurons, with some syllables not eliciting any bursts and one neuron reliably emitting eight bursts for a particularly long and complex syllable.

The principal data set consisted of 115 distinct burst stacks emitted during singing both before and after sleep by 15 RA neurons (seven birds). To compare the stability of temporal structure in premotor activity in the absence of sleep, we also examined the activity of RA neurons recorded in periods of singing and/or calling that did not include sleep. We included in this data set the same 15 neurons used in the sleep analysis, separating out the presleep activity and postsleep activity into distinct sessions, each of which did not include sleep (i.e., 115 burst stacks from presleep recordings, and 115 burst stacks from postsleep recordings, for a total of 230 burst stacks). To expand our data set to include sessions of longer duration without sleep, we included the additional 28 RA neurons recorded from 10 birds (six new, four that were also represented in our sleep-inclusive recordings) in experiments where the lights were not turned out and the birds remained awake throughout, yielding an additional 321 burst stacks. Thus, this "augmented" data set comprised a total of 551 distinct burst stacks recorded from 13 birds.

During one awake-only session, we also briefly recorded one putative RA interneuron characterized by a low baseline firing rate and an especially narrow spike width $(0.13 \mathrm{~ms}$ peak to trough, compared to a range of 0.19-0.41 ms for all other RA neurons we recorded), but we did not include this unit in our analyses because of insufficient spike isolation during singing.

Analysis of burst structure and definition of features and structural changes. To evaluate changes to the temporal structure of premotor bursts across many renditions, we (1) developed a procedure to align all presleep or postsleep bursts for a given burst stack, (2) generated functions that captured the temporal features of the aligned bursts, and (3) evaluated the significance of any temporal or spike count differences between presleep and postsleep groups of spikes.

To optimally align burst renditions within a presleep or postsleep burst stack, we used two procedures: $L_{1}$-distance minimization $\left(L_{1^{-}}\right.$ MIN), as described by Chi and Margoliash (2001), and cross-correlation maximization (CC-MAX). In both cases, the alignment of spike sequences was accomplished by iteratively shifting each burst rendition to either globally minimize the summed $L_{1}$ distances $\left(L_{1}-\mathrm{MIN}\right)$ or maximize summed cross-correlation measures (CC-MAX) across all burst pairs, while preserving each individual burst's interspike intervals. Presleep and postsleep burst stacks were then aligned with each other according to similar procedures, with all of the bursts in each stack shifted as a whole so that the relative timing within each stack was preserved.

The $L_{1}$ metric used in the $L_{1}$-MIN method measures the difference between two spike sequences obtained by averaging over all spikes the temporal difference between each spike and its closest corresponding spike in the other sequence, so that optimal alignment would be achieved by minimizing this measure. To generate a cross-correlation measure for the CC-MAX method, we used the biweight kernel $F(x)=\left[1-(x / D)^{2}\right]^{2}$ for all $|x|<D$, where $D$ is a time window corresponding to the temporal precision of the cross-correlation measure (set to $1.5 \mathrm{~ms}$, a value chosen to approximate the apparent temporal precision of RA premotor spike patterns). The total CC of spike trains $S_{1}, \ldots, S_{k}$ was defined as $\sum_{i<j} K\left(S_{i}\right.$, $\left.S_{j}\right)$, where $K\left(S_{i}, S_{j}\right)=\sum F(s-t)$ over $s$ in $S_{i}$ and $t$ in $S_{j}$. The alignment maximized the total CC by shifting each spike train $S_{i}$ while preserving each individual burst's interspike intervals.

Once bursts within a stack were aligned, fine temporal structure was expressed as the tightly aligned spikes across renditions. A "feature" within a burst was defined as a canonical spike, i.e., a spike produced with reliable timing relative to the other spikes in the burst across many or all renditions. To identify and quantify features, we defined for each group of spike trains an adjusted rate function, $R(t)=\sum_{s \text { in } S} G(|s-t| / D)$, where $s$ is the time of an individual spike within the spike train $S$, and $G(x)=$ $\left(1-x^{2}\right)$ for all $|x|<D$ and 0 for all $|x|>D$ with the predefined time window $D=1.2 \mathrm{~ms}$. (Note that $D=1.2 \mathrm{~ms}$ results in a more precise firing rate estimate than the $1.5 \mathrm{~ms}$ time window used for the original burst alignment, achieving a coarse-to-fine alignment procedure.) We then identified peaks in the rate function. This method captures the changes in features we visually observed but is sensitive to the definition of peaks in the rate function, for example, slight changes in the temporal jitter of a given spike.

For a sample of $N$ presleep spike trains, time $T$ was identified as a feature location if it satisfied four criteria: (1) the averaged adjusted rate function had a local peak at time $T$ [i.e., $r(T) \geq r(s)$ for $s$ between $T \pm D$, where $r(T)=$ mean $(R(T))$ over the sample]; (2) the peak at time $T$ was of significantly high amplitude compared with the variability of the rate function, $\left[r(T) \geq 0.3+t_{N-1}(0.975) \times \sigma(T)\right.$, where $\sigma(T)=\operatorname{SD}(R(T))$ over the sample, and $t_{N-1}$ the inverse $t$-distribution function with $N-1$ degrees of freedom]; (3) the variability of spike times within the predefined time window around $T$ was sufficiently low, $\left[t_{N-1}(0.975) \times \sigma\right.$ $(T) \leq D$, where $\sigma(T)=\mathrm{SD}$ (spike times between $T \pm D$ )]; and (4) the average value of the adjusted rate function on either side of the peak fell off sufficiently quickly such that $|I| \leq 2 \mathrm{~ms}$, where $I$ equals the maximal interval containing $T$ over which $r(s) \geq r(T) / 3$. Under these criteria, $\sim 65 \%$ of all spikes in premotor bursts were identified with located features ( $4.8 \pm 3.1$ total spikes/burst; $3.1 \pm 1.8$ features/burst).

We judged each burst stack as having a "structural change" across the sleep interval if three criteria were met. First, features in the presleep and postsleep adjusted rate functions did not align well. Each feature was evaluated to determine whether we could rule out the existence of a corresponding spike in the corresponding stack (i.e., presleep vs postsleep). If for any feature there was no corresponding feature in the corresponding stack within $0.25 \mathrm{~ms}$, and there was no other peak within $0.5 \mathrm{~ms}$ in the opposite stack's rate function with a magnitude statistically indistinguishable from that of the feature being evaluated, then the burst stack was judged to meet this criterion. Second, there was a statistically significant change in mean spike count of at least 0.5 spikes/burst. This criterion arises from the observed loss (or, rarely, gain) in spikes across sleep intervals (see Results). Third, to reduce the effect of artifacts in alignment, structural changes were flagged only when the first two criteria were satisfied under both $L_{1}$-MIN and CC-MAX alignment procedures. Overall, under both alignment procedures, 37 burst stacks satisfied the first criterion, and 60 satisfied the second, with 33 satisfying both. Thus, changes in spike timing typically were associated with changes in spike rate, but the reverse was not generally the case. Those burst stacks found to undergo structural changes under these criteria 
corresponded well to those burst stacks that appeared to have altered spiking patterns under visual inspection.

Analysis for separator intervals other than sleep. Sleep is a natural separator between groups of vocalizations, but we also explored whether changes to premotor activity occurred at times other than sleep. To this end, for each burst stack we sought to identify the interval between consecutive renditions of bursts that was most likely to correspond to a change in burst structure, referring to the interval thus identified as the "separator interval." We began by measuring the similarity of all possible pairings of individual bursts within each burst stack, using the $L_{1}$ distance metric described above; greater $L_{1}$ distance implies less similarity. Then, we considered each interval between bursts as a candidate separator interval, except that we excluded the first four and last four such intervals to avoid boundary effects. For each candidate interval, we divided the bursts into preinterval and postinterval groups, and from the collection of candidate intervals we identified the one interval that maximized the difference between the mean $L_{1}$ distances of across-group comparisons (preinterval vs postinterval) and within-group comparisons (preinterval vs preinterval, or postinterval vs postinterval). This procedure tended to identify two groups of most-similar bursts, one exclusively before and the other exclusively after the interval, dividing the burst stack at a moment in time that often corresponded to a visibly noticeable change in burst structure (Fig. 1A).

We also used a modified procedure better suited to quantify a subset of the transitions in burst structure. For these cases there was a distinct transition between distinct states, but with one of the states exhibiting less variability than the other. In these cases, the candidate interval that maximized the difference between mean $L_{1}$ distances did not always correspond to the visually observed transition. We found that for these cases, the transition typically coincided with a candidate interval that maximized the differences comparing $L_{1}$ variances for across-group and postinterval (or preinterval) candidate intervals as well as maximizing the differences between $L_{1}$ means for across-group and preinterval (or postinterval) candidate intervals. Therefore, in these cases, we designated the interval thus defined as the separator interval; in all other cases, we simply used the interval maximizing the $L_{1}$-mean distances between across-group and within-group comparisons as the separator interval.

Estimating occurrences of sleep-separator comparisons attributable to chance. Finally, we also developed a statistical procedure to compare the location of separator intervals in recordings that did and did not include sleep. To this end, we first identified separator intervals for the awakeonly recording sessions. Then, for each burst stack, we calculated the proportion of bursts occurring before the separator to the total number of bursts. A histogram of the resulting distribution suggested a quadratic distribution, so we used a quadratic fit to generate a baseline probability density function (PDF) (Fig. 1B).

The PDF estimate allowed us to test the hypothesis that the distribution of $L_{1}$-optimized separator intervals was the same in awake-only and sleep-inclusive recordings. In a bootstrap procedure, we sampled 115 fractions from the PDF and respectively multiplied these by the total number of renditions for each of the 115 burst stacks in our data set to get a random separator interval. We repeated this procedure 10,000 times to obtain a distribution of simulated separator intervals. This distribution was used to evaluate the likelihood that the number of separator intervals we observed to correspond with the period of sleep (either exactly or within one interval) would occur simply by chance.

The quadratic shape of the PDF can be explained as follows. The greater likelihood of locating separator intervals near the endpoint of an experiment rather than in the middle is most likely attributable to the exaggerated effect of outliers on small groups of bursts. The asymmetrical shape of the PDF (see Results) may reflect a slightly increased variability across burst renditions later in experiments, when more time sometimes passed between singing bouts as birds became desensitized to the presence of the adjacent female and tended to sing less frequently.

\section{Results}

We recorded from 43 RA single units while birds vocalized (sang or called; including one "double unit" that we treat equivalently to the single units in our analyses). Only a subset of these record-
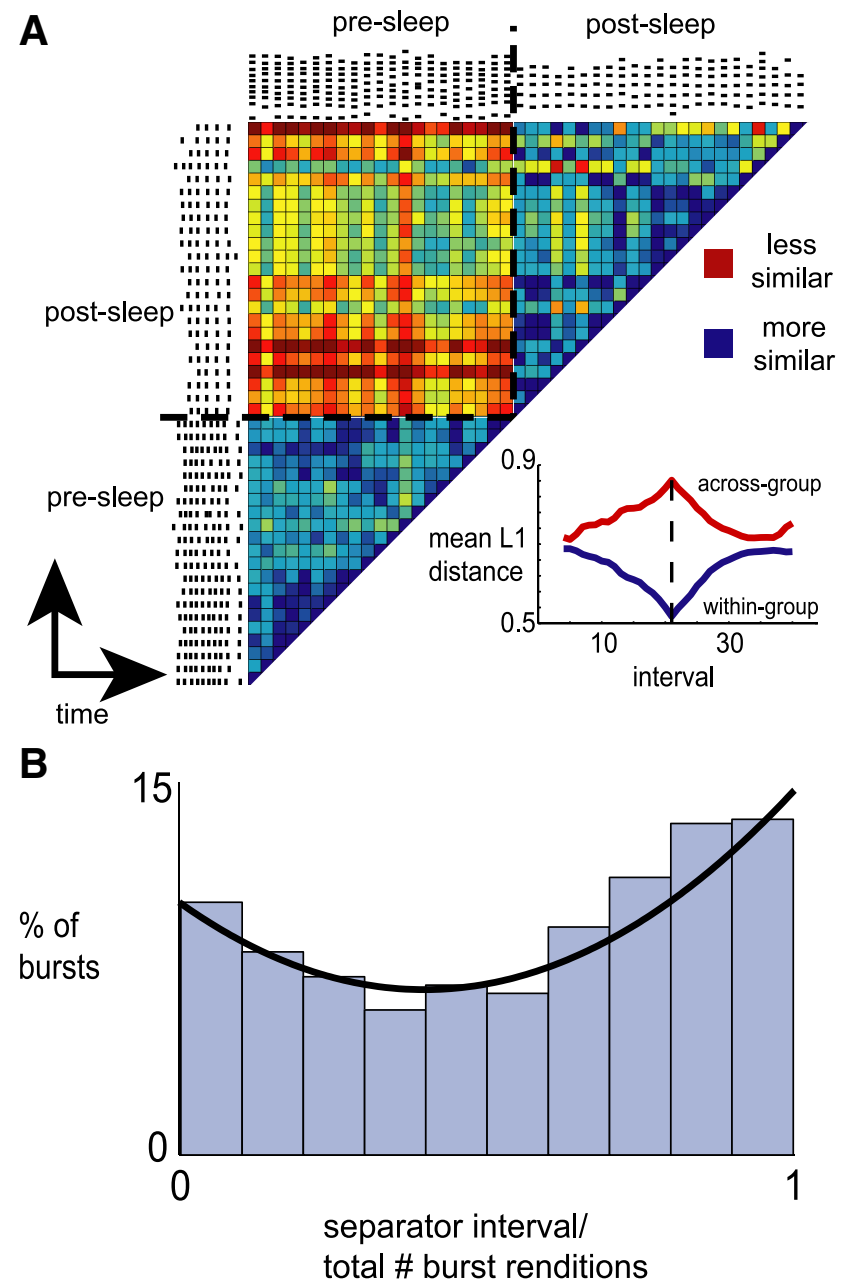

Figure 1. Identifying intervals with possible changes to premotor burst patterns. $A$, Comparisons of pairs of burst renditions for a burst stack recorded before and after a period of sleep, using the $L_{1}$ distance metric. Each row and column represents a single burst rendition (represented by rasters to the left of rows or above columns). Each colored box represents the $L_{1}$ distance between the bursts denoted by the given row and column according to a color map with red indicating the highest $L_{1}$ distances (less similarity) and blue representing the lowest $L_{1}$ distances (more similarity). The sleep interval is denoted by black dashed lines. Note that burst comparisons above and to the left of the sleep-interval lines (i.e., comparisons between presleep and postsleep bursts) show less similarity than do comparisons between bursts taking place exclusively before or after sleep. The graph at the bottom right shows the mean $L_{1}$ distances between all pairs of burst renditions taking place across the separator interval (red line) or exclusively before or after the separator interval (blue line) for all possible intervals. The sleep interval is denoted by the dashed line, where the difference in mean $L_{1}$ distance between these two groups reaches a clear peak; such a peak defines the optimized separator interval. $\boldsymbol{B}$, Estimated probability distribution function for the location of the optimized separator interval in recording sessions that did not include sleep ( 551 burst stacks). The histogram shows the distribution of optimized separator intervals relative to the total number of burst renditions within each recording session. A quadratic fit (line) was used to determine the PDF.

ings was maintained through a period of sleep and subsequent vocalizations (Table 1) (see Materials and Methods). It is likely that all of these cells were projection neurons targeting the brainstem, given their fast $(>30 \mathrm{~Hz})$, regular baseline spiking activity and bursting activity during singing (Spiro et al., 1999; Leonardo and Fee, 2005). Each cell reliably burst with consistent timing relative to specific vocalizations such as a particular syllable or call; thus, raster plots of the neuronal activity aligned to vocalization onsets produced "stacks" of bursts, which were the basis for our analysis (see Materials and Methods). In the 37 cells for which we recorded singing, there were $10.1 \pm 4.2$ unique burst stacks 
Table 1. Distribution of recordings across different experimental conditions

\begin{tabular}{lccc}
\hline Recording session type & Birds & Neurons & Burst stacks \\
\hline Sleep inclusive (all) & 7 & 15 & 115 \\
Sleep inclusive (short) & 4 & $10^{b}$ & 83 \\
Sleep inclusive (long) $^{\text {No sleep }}{ }^{a}$ & 3 & 5 & 32 \\
\hline
\end{tabular}

${ }^{a}$ Includes both the presleep-only and postsleep-only portions of the sleep-inclusive sessions as distinct no-sleep sessions.

"Includes one "double unit" counted as a single neuron in our analyses.

per song, and $1.9 \pm 1.1$ unique burst stacks per call. For the remaining six cells for which we only recorded calls, there were one to two unique burst stacks per call.

We examined the effects of sleep on vocalization-related neural activity in adult male zebra finches under two protocols: short, interrupted periods of sleep and full, uninterrupted nights of sleep. The distribution of recording sessions according to experimental protocol is reported in Table 1. Birds in the short-sleep design ( $n=10$ neurons in 4 birds) experienced a period of darkness lasting 90-179 $\mathrm{min}$ (average, $136 \pm 31 \mathrm{~min}$ ), whereas the birds in the second design ( $n=5$ neurons in 3 birds) experienced a full $8-10 \mathrm{~h}$ of darkness. For all birds, during the first $2-10 \mathrm{~min}$ of darkness, birds typically rapidly transitioned between short periods of wake and sleep. Initially, in some cases, sudden awakenings apparently resulted from playback of the bird's own song that was presented during short-sleep sessions (see Materials and Methods), but birds quickly habituated to the song playback and began to reliably sleep through the stimulus. Birds were judged to begin an extended period of sleep when a full minute passed with no two consecutive 3 s intervals classified as "awake" (see Materials and Methods) (see supplemental Fig. 1, available at www. jneurosci.org as supplemental material). Based on the measure of spontaneous activity, the onset of extended periods of sleep began $10.5 \pm 8.8 \mathrm{~min}$ (range, 1.3-29.5 $\mathrm{min}$ ) after the start of subjective night, and represented $78.8 \pm 10.5 \%$ (range $67.8-94.9 \%$ ) of the total dark phase after sleep onset. Thus, whereas the recording situation probably disrupted the animal's total sleep and sleep architecture to some degree, each animal experienced a considerable amount of sleep including extended periods of uninterrupted sleep.

\section{Premotor bursts change after sleep}

The structure of RA premotor bursts is highly conserved in songs directed toward females, as a bird repeats the same stereotyped syllable within and across songs (Yu and Margoliash, 1996). We frequently observed, however, changes to the structure of these bursts after sleep. The only systematic change across time described previously in RA premotor bursts is the submillisecond magnitude temporal drift in the timing between bursts (Chi and Margoliash, 2001). In contrast, in the present data, many clear and persistent changes in premotor patterns associated with the sleep interval were apparent by visual inspection beginning with the first song renditions after sleep; these were marked by the elimination or, rarely, addition of spikes in the postsleep pattern (Fig. 2). In cases where bursts had fewer spikes after sleep, the decrease in spike number was often accompanied by an increase in interspike interval (Fig. 2A,B). Typically it was difficult or impossible to identify a specific spike from the presleep bursts that was eliminated after sleep. Instead we saw a restructuring of the entire burst.

Once a change occurred, it tended to be stable. Clear changes to burst structure after full nights of sleep were obvious in many cases. The changes persisted for as long as we could hold the recording - in one case, for several hours after waking (Fig. 3). Whatever the cellular or network effects that led to these changes, they achieved their suprathreshold effects during sleep or immediately after awakening, and persisted thereafter.

To quantitatively assess these changes in burst structure, we aligned all presleep and postsleep bursts for each of the 115 burst stacks using two algorithms $-L_{1}$-distance minimization and cross-correlation maximization-converted these into probabilistic rate functions, located features within those functions, and identified reliable changes in features, which we call "structural changes" (see Materials and Methods). Using these criteria, we found that for recordings spanning a sleep interval, 33 of 115 burst stacks showed structural changes (Fig. 4). These 33 burst stacks were distributed across 10 of 15 neurons recorded across sleep, with each neuron exhibiting one (four neurons), two (three neurons), or six (two neurons) bursts with structural changes, but with one neuron exhibiting 11 bursts with structural changes. The statistical significance of all the results that follow was maintained even with the neuron with 11 bursts with structural changes removed.

Considering the sequence of syllables within motifs or the sequence of bursts within a syllable, there was no apparent tendency for structural changes to be associated with bursts that occurred in any particular syllable within the motif, or within any particular burst within a syllable. There was also no clear difference in the number of changes in burst stacks associated with contact calls ( 5 of $21 ; 24 \%$ ) compared to those associated with song syllables ( 28 of $94 ; 30 \% ; p=0.79$, Fisher's exact test).

We tested the hypothesis that the two different experimental designs affected the rate of occurrence of structural changes. There were no significant differences in the frequency of burst changes between short-sleep and long-sleep birds. The frequencies of structural changes [27\% (22 of 83) short sleep, 34\% (11 of 32) long sleep; $p=0.40 ; \chi^{2}=0.70$ ] were similar under both experimental conditions, suggesting that the truncated period of sleep and the presence of auditory stimulation were not significant factors in driving premotor plasticity in RA neurons.

Although rare, there were also examples of structural changes that occurred during the subjective day. For this analysis, we used an augmented data set including a number of daytime-only recordings (see Materials and Methods). To quantitatively assess the rate of structural changes while the birds were awake, we simply chose the longest vocalization-free interval with a sufficient number of vocalizations $(n \geq 8)$ both preceding and following the interval, and compared the bursts before and after this interval. Across 551 distinct burst stacks in the augmented data set (43 neurons, 13 birds; see Materials and Methods) (see Table 1 ), the rate of structural changes that occurred in recordings that did not span a sleep interval was much lower $(3.3 \% ; 18$ of 551$)$ than the rate of changes across the sleep interval $(28.7 \%$; 33 of $115)$, and this difference was significant $\left(p<0.001 ; \chi^{2}=87.0\right)$.

Finally, since the recordings that included a period of sleep were generally the ones of greatest duration, we also tested whether the more frequent occurrences of changes to burst patterns in these recordings resulted from the additional passage of time rather than the presence of sleep. We selected the longest (100-360 $\mathrm{min})$ awake-only recording sessions $(n=8$, with 71 distinct burst classes). For each of these recordings, we defined an artificial separation interval $(1.5-3 \mathrm{~h})$ of similar duration to the dark periods in the shorter-sleep recording sessions and then compared the two sets of recordings. Even when controlling for 
the passage of time, burst patterns underwent many more changes across sleep than across wakefulness. During short sleep-inclusive recording sessions, approximately one-fourth (22 of 83 ) of bursts exhibited structural changes, most of which were obvious under visual inspection. In the recordings with the imposed artificial separation interval, no structural changes were obvious under visual inspection, and a much lower number of bursts ( 4 of $71 ; 6 \% ; p<0.001$, Fisher's exact test) exhibited structural changes.

Changes in premotor activity occur across sleep-inclusive intervals

The preceding results show that using sleep as the separator interval reliably identifies changes in RA burst patterns, but this does not rule out the possibility that the changes actually tended to occur just before sleep (which could occur if the bird could anticipate the onset of the sleep period) or just after sleep. We formulated this hypothesis rigorously as a test of whether there are previously undetected changes to premotor burst patterns expressed as a transition between distinct states, in which a previously stable temporal spiking pattern is replaced with a different pattern which then persists throughout the subsequent songs. We then compared those transitions to the occurrence of sleep.

To test this hypothesis, for each of the 115 distinct burst stacks associated with the 15 neurons, we identified a separator interval using an algorithm designed to identify the interval most likely to represent a transition to an altered burst pattern (see Materials and Methods), ignoring when sleep actually occurred. A large proportion of the separator intervals thus identified occurred close to sleep: 30 of 115 separator intervals (seven neurons, four birds) either coincided with sleep or fell between the last two bursts before sleep or the first two bursts after sleep. To assess whether the degree of coincidence between the separator intervals and sleep was attributable to chance, we generated predictions of the underlying distribution of separator intervals using the premotor activity of RA neurons recorded in the contiguous periods that did not include sleep (see Materials and Methods). We then randomly sampled from the predicted distribution 10,000 times for each of the set of 115 burst stacks to estimate the distributions of separator intervals predicted by chance if the presence of sleep did not bias the locations of the separator intervals.

Overall, in the simulated data, the average number of exact matches between separator intervals and sleep was $2.5 \pm 1.6$ burst stacks (of 115 total), with a maximum of 11 such matches-just half of the 22 exact matches between separator intervals and sleep

C
A
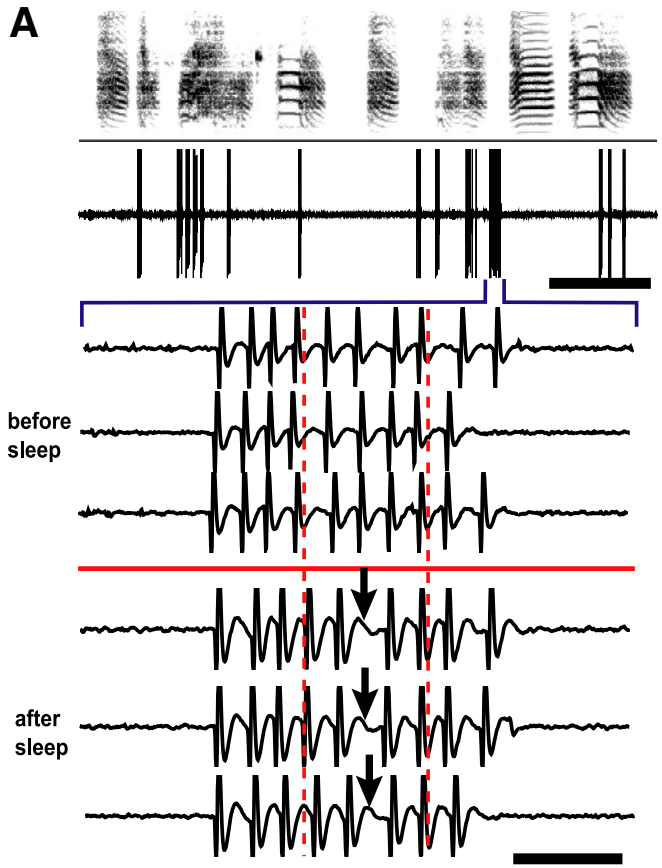

B

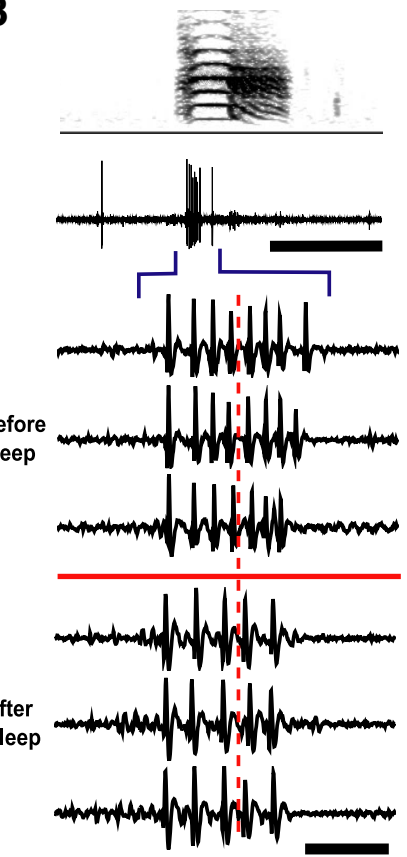

Figure 2. Changes to temporal structure of RA premotor bursts across a period of sleep. $A$, Recordings from an RA neuron that produced fewer spikes in premotor bursts associated with a song syllable after a $2 \mathrm{~h}$ sleep period. Top, Spectrograph of song aligned with simultaneous recording of premotor neuronal activity. Middle, Recordings of neuronal activity during three renditions of the song syllable. Bottom, Neuronal activity during three more renditions of the same song syllable after sleep. The leftmost vertical dashed line follows the fourth spike in all presleep bursts but precedes the fourth spike in all postsleep bursts, and the rightmost dashed line does the same with the eighth spikes. Note that whereas both presleep and postsleep bursts inconsistently include an extra spike at the end, the postsleep bursts consistently produce one spike fewer than presleep bursts, with an accompanying gap in the middle of each burst (arrows). $\boldsymbol{B}$, Recordings from another RA neuron that produced fewer premotor spikes during production of a contact call after a $2.5 \mathrm{~h}$ sleep period. The dashed line separates fourth spikes in each burst as per $\boldsymbol{A}$. $\boldsymbol{C}$, Recordings from a third RA neuron that showed extra spikes in two premotor bursts associated with a song syllable after a $2 \mathrm{~h}$ sleep period. Scale bars: $A$, top, $250 \mathrm{~ms}$; bottom, $10 \mathrm{~ms}$; $\boldsymbol{B}$, top, $100 \mathrm{~ms}$; bottom, $10 \mathrm{~ms}$; $\boldsymbol{C}$, top, $300 \mathrm{~ms}$; bottom, $25 \mathrm{~ms}$.

we observed in our actual recordings. Furthermore, the same sampling procedure applied to the 93 burst stacks not showing exact matches between separator intervals and sleep yielded an average of $3.8 \pm 1.9$ examples of separator intervals occurring within \pm 1 interval of sleep. In contrast, there were eight such examples in the actual data, and only $3.7 \%$ of the random trials had eight or more such examples (Fig. 5A). In total, the 30 sleepseparator coincidences (exact or within one interval) we observed in the actual data set were much more than was ever observed in the simulated data (maximum, 17). These distinctions were also 

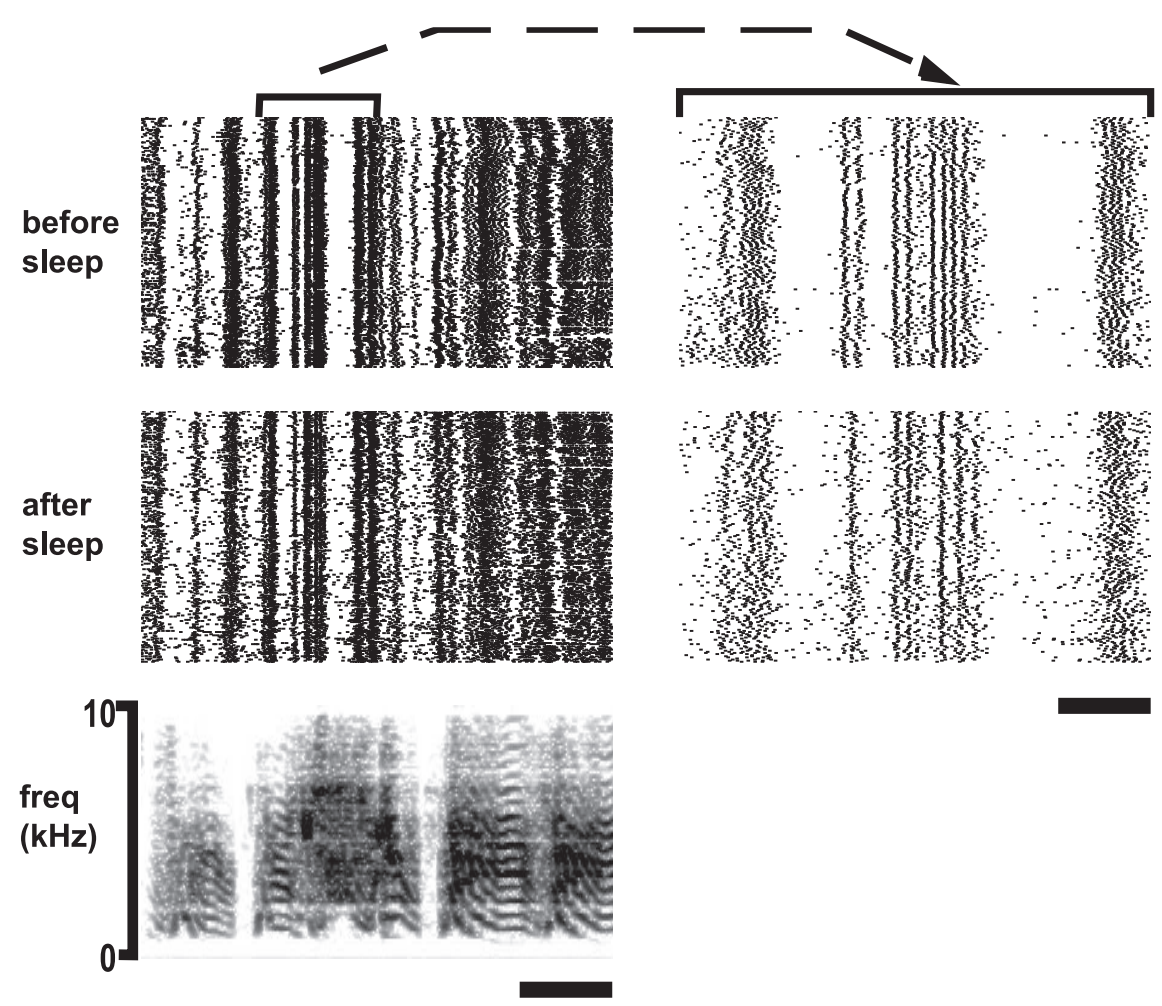

Figure 3. Persistent structural change to premotor bursts in a pair of RA neurons after a full night of sleep. Left, Raster plots of spiking activity during production of the song motif before (top) and after (middle) sleep. This site was the "double unit" described in the text, in which the activity of a pair of neurons could reliably be distinguished from the background activity but not from each other; the data were treated equivalently to single-unit data. Note the stability of the burst patterns within the before-sleep and after-sleep groups, even over long periods of time (before sleep, 171 min; after sleep, $459 \mathrm{~min}$ ). Rasters are aligned within each group using the $L_{1}$-minimization method, and the groups are aligned with a spectrograph of the song motif (bottom). Right, Finer temporal detail. The persistent loss of spikes from the middle bursts is clear, and the temporal pattern of the bursts fails to return to the presleep pattern even after several hours of postsleep singing. Scale bars: Left, $100 \mathrm{~ms}$; right, $25 \mathrm{~ms}$.

maintained when assessing coincidence between the separator interval and sleep with a $30 \mathrm{~s}$ criterion (Fig. 5B). A similar analysis comparing short-sleep recording sessions to awake-only recording sessions of similar duration confirmed this result (see supplemental material available at www.jneurosci.org). Finally, the distribution of separator intervals drawn from the actual data that did not coincide with sleep (i.e., the 85 of 115 separator intervals differing from sleep by at least two intervals) did not exhibit significant difference from the corresponding simulated distribution ( $p=0.14$, Kolmogorov-Smirnov test). Considered together, the overall distribution of separator intervals drawn from the actual data was significantly different from the simulation distribution $(p<0.01$, Kolmogorov-Smirnov test $)$.

We note that our technique for identifying separator intervals depends on statistical comparisons of the $L_{1}$ measure across populations of bursts, and therefore the estimates of the timing of putative changes to burst structure will exhibit noise that arises from rendition-to-rendition variability of premotor bursts in RA neurons. In fact, simply using sleep as an indicator for possible changes proved a more effective strategy for identifying structural changes, as only 27 of 115 burst classes exhibited such changes across algorithm-identified separator intervals, compared with the 33 changes observed across sleep. Since sleep was more reliable for locating structural changes than was the algorithmic approach ignoring sleep, this supports the conclusion that under the conditions of our experiment, sleep itself induced discrete changes in premotor activity.

\section{Structural changes tend to cluster across song}

In those neurons exhibiting structural changes in multiple burst stacks, we observed that changed bursts had a significant tendency to cluster together. Across the five neurons with changes to more than one burst stack within the song motif, the spread of changed bursts (i.e., the interval between the first and last changed bursts) covered an average of $61 \%$ of the total number of bursts within each motif. However, given the number of changed bursts for each neuron, a random distribution of burst changes (determined by shuffling the distribution of changes within each neuron 100,000 times) would be expected to yield an average spread of $74 \pm 7 \%$ of the total, and spreads as low as or lower than the observed $61 \%$ occurred in only $1.9 \%$ of the shuffled trials. The clustering was even more striking when excluding the neuron in which nearly every burst changed: in this restricted data set, the average spread between the first and last changed bursts covered $53 \%$ of the total number of bursts. This compares to an expected coverage of $69 \pm 9 \%$ in the corresponding shuffled trials, with only $1.6 \%$ of shuffled trials showing coverage as low as actually observed. Across all neurons with multiple burst changes, nearly one-third (6 of 19) of all intervals between changed bursts included an unaltered burst. Overall, these data indicate that the mechanism of change was biased to act over temporally restricted portions of the motor program.

\section{Loss of spikes after sleep}

We now turn to describing the changes in the structure of spike bursts in singing before and after sleep. A striking characteristic of singing after sleep was the widespread reduction of overall spiking activity of RA neurons. Most (28 of 33) of the structural changes were characterized by a loss of spikes (average loss of $1.48 \pm 0.98$ spikes/burst; range, $0.55-4.70$ ), with the remaining five changed burst stacks gaining spikes (average gain of $1.07 \pm$ 0.62 spikes/burst; range, $0.56-2.10$ ). Across all burst classes with structural changes, there was an average loss of $1.10 \pm 1.31$ spikes/burst (representing $16.7 \pm 19.9 \%$ of the total number of presleep spikes/burst). The burst durations did not decrease significantly however (average change $-0.20 \pm 4.18 \mathrm{~ms} ; p=0.79$, paired $t$ test), because spike loss was associated with a significant average increase of $0.55 \pm 1.22 \mathrm{~ms}$ in the interspike intervals $(p<$ 0.05 , paired $t$ test comparing bursts before and after sleep; both burst durations and spike intervals were normally distributed).

In contrast to changes in firing rates after sleep, spike loss was not clearly present in structural changes during daytime singing. A small majority of such burst stacks (11 of 18) showed reduction in spike number, with an average change across all 18 of these burst classes of $-0.39 \pm 1.10$ spikes/burst, a statistically insignificant change ( $p=0.15, t$ test). This average change represented an overall loss of only $4.4 \%$ of the total number of spikes for these bursts. Thus, there is some tendency in daytime recordings for 

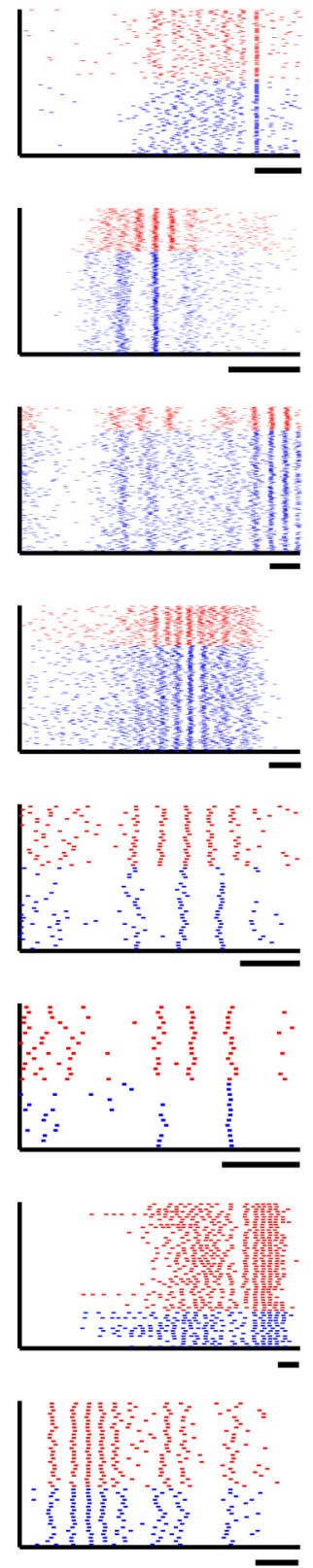
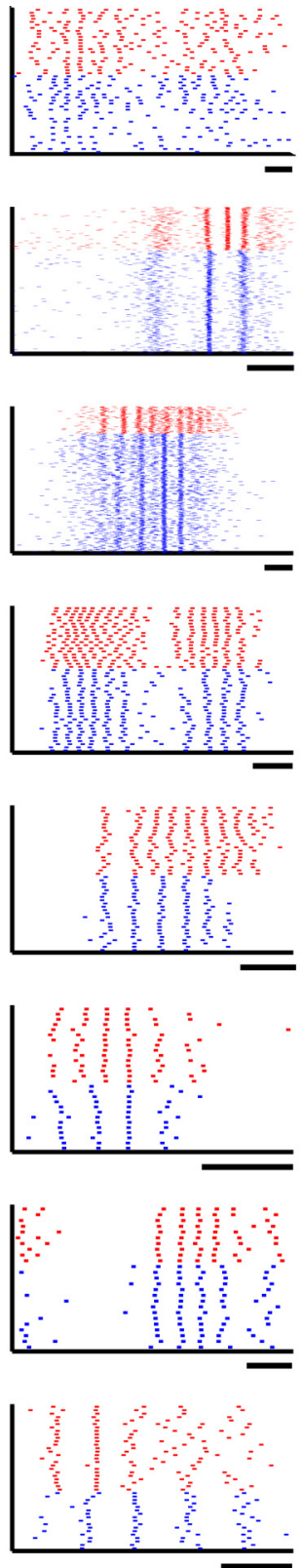
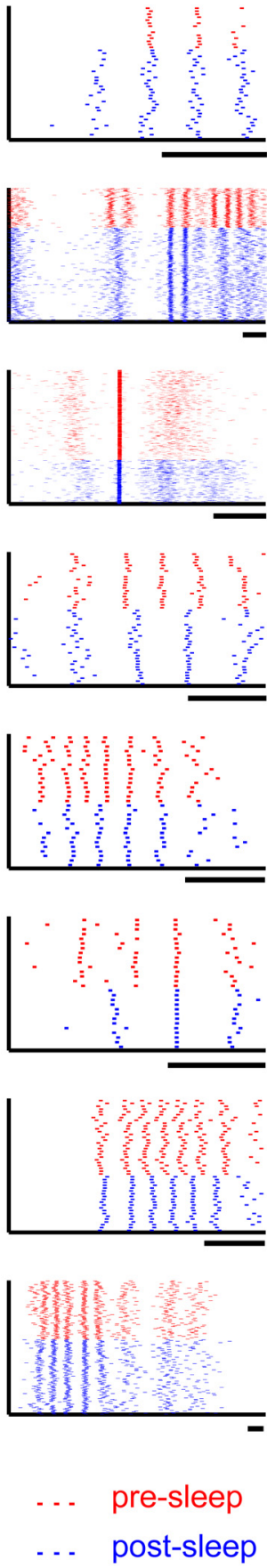
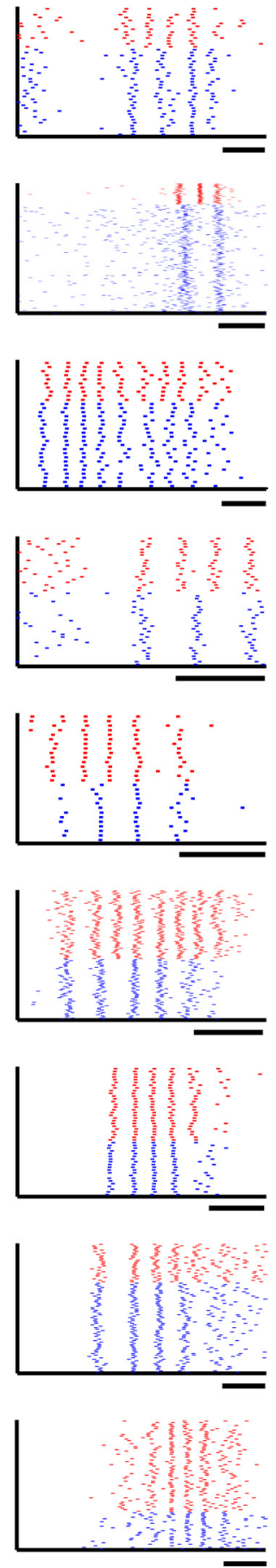

Figure 4. Changes to premotor burst structure in RA neurons. Raster plots for 33 premotor bursts that exhibited structural changes across sleep are shown. Presleep rasters are in red, and postsleep rasters are in blue. Scale bars, $5 \mathrm{~ms}$.

the same phenomenon as observed across sleep, but it is much weaker.

The preceding analyses were restricted to bursts exhibiting structural changes; we now broaden consideration to all bursts. When considering all bursts, there was a significant net loss of spikes after sleep $(0.30 \pm 0.97$ spikes/burst; $p<0.01)$, resulting in an overall reduction of $6 \%$ from presleep spike counts. This apparent global reduction, however, was entirely accounted for by the spike losses observed in conjunction with structural changes. There was no significant tendency for bursts without structural changes to gain or lose spikes (average net increase, $0.02 \pm 0.52$ spikes/burst, representing $0.5 \pm 12.9 \%$ of presleep spikes/burst; $p=0.75$, paired $t$ test).

Bursts that had not undergone structural changes nevertheless had a strong tendency to show changes in spike counts after sleep. Many bursts exhibited a change in the tendency for a given spike to occur (often apparently the last spike), or for a change in the rate of activity in more variable sections of the bursts. Such changes were not well characterized by the feature-based analysis but resulted in spike count changes. Comparing mean spike counts of presleep burst renditions with mean spike counts of postsleep burst renditions, we observed that $45 \%$ (37 of 82 ) of 

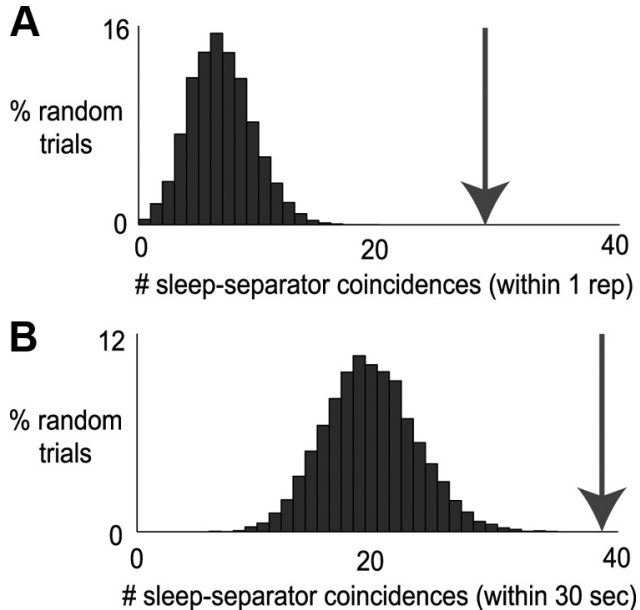

Figure 5. Bias of $L_{1}$-optimized separators toward sleep-inclusive intervals. $\boldsymbol{A}$, Histogram of the distribution of separator-sleep coincidences (defined as zero- or one-syllable renditions between the $L_{1}$-optimized separator and dark phase) generated from 10,000 randomly determined separators drawn for each burst class from the PDF shown in Figure $1 B$. The 30 of 115 coincidences actually observed in the data (arrow) were much more than the mean number predicted by chance alone. $\boldsymbol{B}$, Histogram of separator-sleep coincidences according to wallclock time (defined as $<30$ s between the $L_{1}$-optimized separator and dark phase) generated from the same 10,000 randomly determined sets of separators used in $\boldsymbol{A}$. The 39 of 115 examples observed in the data (arrow) were more than was ever observed under random sampling.

stronger across sleep than in daytime recordings, where spike counts were significantly more stable: only $17 \%$ of all burst stacks (95 of 551) had significant spike count differences across the daytime separator intervals used for our prior analyses $(p<$ $\left.0.001 ; \chi^{2}=38.0\right)$. The mean magnitude of change was also greater across sleep $(0.69 \pm 0.84$ spikes/burst $)$ than across waking periods of similar duration $(0.38 \pm 0.46$ spikes/burst; $p<$ $0.01, t$ test).

We conclude that there is an ongoing process of increases and decreases in spike counts of RA bursts, but only over the sleep interval are these changes strongly biased toward spike loss and decreasing spike counts. Spike loss must be compensated for by an as yet unidentified regenerative process that increases spike counts (see Discussion).

\section{Temporary shifts in burst timing after sleep}

Finally, we observed one additional form of neuronal variability. The period immediately after birds awoke was occasionally associated with a temporary increase in duration of intervals between bursts within syllables. This was observed for 14 of 55 interburst intervals ( 10 of 31 syllables, 4 of 12 neurons), with all shifts tending toward longer intervals (average increase, $3.54 \pm 2.24 \mathrm{~ms}$ ), and the magnitude of the shift representing $10.2 \pm 9.4 \%$ of the mean presleep duration of that interval. In contrast, only one burst interval exhibited a significant change during singing that immediately preceded the subjective night, a decrease of $0.81 \mathrm{~ms}(8.7 \%)$ from the average preceding interval. In most (10 of 14) cases, interburst intervals rapidly recovered to presleep values. The period of relaxation to presleep intervals was $323 \pm$ 370 s.

The appearance of changes in interburst timing was not correlated with the appearance of changes in burst structure. Bursts bordering intervals with significant postsleep drift had similar frequency of structural changes $(25 \%$; 6 of 24$)$ compared with other bursts (structural changes 27 of 91; 30\%; $p=0.80$, Fisher's exact test). This observation indicates that changes to burst structure are not the result of changes to song tempo expressed at the timescale of interburst interval plasticity.

Particularly favorable examples were cases of changes in interburst intervals that were observed for long calls, which birds often produce within seconds upon waking, whereas even in favorable cases they only begin to sing minutes later. Four of these cases (out of seven total interburst intervals, three sites, two birds) represent dramatic examples of plasticity immediately after wakening (Fig. 6). In these cases, many (18-115) long calls were produced starting 15-97 s after waking and continuing for 141-542 s preceding the first postsleep song. The overall magnitude of change in spike timing at the onset

Figure 6. Temporary postsleep plasticity in interburst timing during calling. Left, Raster plots of premotor activity at three sites (three separate nights) in one bird during production of distance calls. Note that the activity after sleep (below the horizontal dashed lines) initially shows greater interburst intervals, which then gradually relax toward the presleep values. Right, Raster plots of premotor activity at the same site during production of selected song syllables. Brackets indicate periods of overlapping singing and calling activity after sleep; note that the postsleep increase in interburst intervals during call production has mostly vanished by the time singing commences. Scale bar, $20 \mathrm{~ms}$.

burst stacks not exhibiting structural changes nevertheless underwent significant changes (unpaired $t$ test) in their mean spike counts. There was no bias toward loss or gain of spikes. The overall tendency for spike counts to change was significantly of the light phase was $6.2 \pm 2.2 \mathrm{~ms}$. By the time singing commenced, interburst intervals associated with long calls had already returned to presleep durations. Burst intervals associated with song syllables recorded at those same sites exhibited only 
modest increases of 1-3 ms followed by gradual change toward presleep interval durations ( 7 intervals) or no significant changes (14 intervals). These data help to emphasize that the process underlying structural changes proximate to sleep produced stable changes that are distinct from the temporary plasticity manifest in the period immediately after wakening. The song and burst timing variability suggest that adult zebra finches exhibit a brief period of performance variability immediately after waking that is akin to performance variability termed sleep inertia in humans (Dinges, 1990). The period of sleep inertia is shorter in finches than in humans (Jewett et al., 1999).

\section{Discussion}

We demonstrated that periods of sleep are commonly associated with small but secure changes in the burst structure of RA neurons. RA burst changes occurred even when sleep was artificially curtailed, indicating that the process of change is related to sleep itself and is not a manifestation of circadian cycles. This also argues against nonspecific effects (such as movement artifacts or electrochemical changes at the electrode tip associated with longduration recordings) driving the observed changes in burst structure. Nonspecific effects should increase monotonically with the amount of movement (most prominent during daytime singing) or duration of recordings, but we observed no such correlations.

The spontaneously tonically firing neurons we recorded from are qualitatively similar to the presumptive RA projection neurons (RAps) recorded during singing but held for shorter periods of time (Yu and Margoliash, 1996; Dave and Margoliash, 2000; Leonardo and Fee, 2005). In contrast, RA interneurons are likely to fire more sporadically (Spiro et al., 1999; Leonardo and Fee, 2005) (see Materials and Methods) and are very rarely isolated in chronic recordings. Our experimental design was challengingmaintaining recordings over two singing periods separated by sleep. Our sample size is correspondingly small, collected over several years of recordings. If our sample of RAps is unbiased, this implies that approximately half of all RA projection neurons alter their burst patterns on a nightly basis, and that these changes are expressed in $>40 \%$ of the bursts in those neurons.

The nucleus RA represents the sole forebrain output of the song system, projecting to brainstem nuclei that control the syrinx and regulate respiratory rhythm. Sparse singing activity in RA-projecting HVC neurons (HVC-RAns) probably represents a time code (Hahnloser et al., 2002; Long and Fee, 2008), which is converted to a denser representation representing notes (parts of syllables) in RA (Yu and Margoliash, 1996; Leonardo and Fee, 2005). Accumulated continuously throughout adult life, it seems unlikely that the observed level of RA nocturnal spike loss could represent uncompensated noise or drift without changes in syllable morphology or larger circadian changes in adult song than have been observed (Derégnaucourt et al., 2005; Glaze and Troyer, 2006). Instead, we hypothesize that RAp nocturnal variation is a component of adult song maintenance, an active process mediated by auditory feedback (Nordeen and Nordeen, 1992; Leonardo and Konishi, 1999; Andalman and Fee, 2009; Sober and Brainard, 2009). The apparent role of nighttime sleep in song maintenance as revealed by song deterioration after adult deafening is consistent with this hypothesis (Derégnaucourt et al., 2005). RA bursting during sleep adaptively changes with developmental song learning, providing additional if indirect support for this hypothesis (Shank and Margoliash, 2009). In songbirds, syringeal muscles are "superfast," exhibiting functional modulation at frequencies exceeding $200 \mathrm{~Hz}$ (Elemans et al., 2008), and could be sensitive to changes distributed over a population of
RAps at the very fine time scale we observed for single neurons. Information carried by neuronal replay could serve as the substrate for fine-tuning neural networks during sleep, where the spontaneous replay of premotor bursts has been observed in RA (Dave and Margoliash, 2000) and may play similar roles in other systems (Wilson and McNaughton, 1994; Qin et al., 1997; Nadasdy et al., 1999; Hoffman and McNaughton, 2002; Pennartz et al., 2004; Ji and Wilson, 2007; Peyrache et al., 2009).

\section{Circuit models of RA burst changes}

It is helpful to consider models of how RA bursts are generated. Sleep-mediated changes in burst structure could reflect changes in inputs to RA and/or changes in RA local circuits. During sleep, spontaneous RA activity is strongly driven by HVC activity (Dave and Margoliash, 2000; Hahnloser et al., 2006). HVC-RAns have been described as sparsely firing, each neuron emitting a highly regulated single short burst once per motif (Hahnloser et al., 2002; Kozhevnikov and Fee, 2007). A recent "clock" model posits that RAp activity is dominated by clock-like, feedforward excitation from HVC (Leonardo and Fee, 2005), with a given population of HVC-RAns broadly distributed in HVC projecting onto single RAps, and a subset of HVC-RAns active at any moment in time. Changing one or a few HVC-RAns, representing a particular time point in the clock, could affect a specific burst of an RA neuron.

A second model of RA bursting arises from the observation that each RAp spontaneously oscillates at a given frequency ( $\mathrm{Yu}$ and Margoliash, 1996), which arises from the interaction of intrinsic RAp subthreshold oscillations with network properties (Mooney, 1992). The "reconfiguration" model posits that during singing, different RA neurons (viewed as simple oscillators) are dynamically coupled and uncoupled by the action of interneurons, resulting in transient local networks with complex bursting during singing such as is actually observed for RA neurons. In this model, HVC inputs would select different local RA circuits via changes in the synaptic weights onto RAps, thereby influencing RA burst patterns. Long-distance inhibitory interactions within RA could help support rapid coupling of different sets of RA projection neurons (Spiro et al., 1999). The principal distinction from the clock model of Leonardo and Fee (2005) is that local RA circuits contribute to the structure and the timing of RA bursts. We note that the two models are not mutually exclusive, and alternate models may obtain (Trevisan et al., 2006). Additionally, neither model considers a recently described class of RAps that is reciprocally connected with HVC (Roberts et al., 2008).

To directly compare the two models, we consider the case where the spike loss we observed arises from loss of synaptic drive from HVC-RAns onto RAps. (Alternatively, spike loss could arise from changes local to RA.) How would reduction in HVC drive manifest as variation in RA bursting? Structural changes were characterized by spike loss, not changes in burst timing. If several HVC-RAns represent any given moment in time, this is consistent with the clock model, so that loss of some HVC-RAn input changes the magnitude but not the timing of the drive into an RAp. Spike loss was also associated with compensatory changes in interspike intervals tending to affect the entire burst, yet HVC-RAn bursts are much shorter than the duration of many RAp bursts. Either the timing of HVC-RAns "tile" the duration of an RAp burst and several HVC-RAns (representing nonidentical points in time) change, or several simultaneous HVC-RAn inputs (one or more of which is lost) initiate an RAp burst but do not regulate it thereafter. 
Models of RA bursting also need to account for the distribution of bursts that changed in a given RAp. The percentage of neurons with changed bursts decreased monotonically from zero changed bursts per neuron to the one cell with 11 changed bursts, and there was a tendency for changed bursts to be temporally restricted in song but not necessarily temporally contiguous. For the clock model, temporal contiguity of changes could result if any plastic event in HVC that occurs during sleep were to propagate down the chain of HVC-RAns associated with that RAp. To fit the observed data, it would also be necessary for the plastic changes to skip some HVC-RAns while continuing to propagate down the chain. For the reconfiguration model, an attractive hypothesis is that during sleep a small number of RAps exhibit robust changes in somatic or proximate dendrite conductances (e.g., the RAp with most of its bursts changed), and that the effect on other RAps depend on how many circuits the two share throughout song. To account for the temporal contiguity of the burst changes requires another assumption, most parsimoniously that nearby RA neurons tend to participate in more shared circuits.

\section{Homeostatic regulation of electrical excitability}

Spike elimination during sleep must be compensated by spike gain, otherwise over time RAps would cease firing. Spike gain could occur during the day, for example during undirected singing not sampled in our experiments. Undirected singing is characterized by increased temporal variability in acoustic output (Kao et al., 2005; Olveczky et al., 2005), and greater variability in activity of the neurons of the lateral magnocellular nucleus of the nidopallium (LMAN) (Hessler and Doupe, 1999; Leonardo, 2004) that help regulate auditory-feedback-mediated song maintenance (Brainard and Doupe, 2000). Adult neurogenesis is prominent in the population of HVC-RAns (Alvarez-Buylla and Kirn, 1997; Scott and Lois, 2007; Scotto-Lomassese et al., 2007). Incorporation of new functional synapses could increase excitability of RA neurons. By this scenario, RA neurons could lose excitatory input over multiple nights and then suddenly regain it (presumably at night). The latter, hypothesized event would be rare, and difficult to detect in electrophysiological recordings.

The mechanisms of spike loss, and presumptive spike gain, may be specific to the song system, but regulation of levels of spiking activity may be a more general phenomenon. It has been proposed that homeostatic mechanisms regulate the overall level of excitation in the nervous system (Turrigiano and Nelson, 2004). Spike loss during sleep could be a superthreshold manifestation of such mechanisms, shedding excess spikes that accumulate during daytime activity (Vyazovskiy et al., 2009) to maintain a static level of overall network excitability, and is consistent with recent evidence for a net potentiation of synaptic strength in rat cortex and hippocampus during wakefulness and net depression during sleep (Vyazovskiy et al., 2008). If spike loss of the same magnitude as we have observed in RAps occurs in other systems, it may be difficult to detect with single-cell recordings in those systems, where spiking activity is less precise than that of RAps.

\section{References}

Alvarez-Buylla A, Kirn JR (1997) Birth, migration, incorporation, and death of vocal control neurons in adult songbirds. J Neurobiol 33:585-601.

Andalman AS, Fee MS (2009) A basal ganglia-forebrain circuit in the songbird biases motor output to avoid vocal errors. Proc Natl Acad Sci U S A 106:12518-12523.

Aton SJ, Seibt J, Dumoulin M, Jha SK, Steinmetz N, Coleman T, Naidoo N, Frank MG (2009) Mechanisms of sleep-dependent consolidation of cortical plasticity. Neuron 61:454-466.
Brainard MS, Doupe AJ (2000) Auditory feedback in learning and maintenance of vocal behaviour. Nat Rev Neurosci 1:31-40.

Brawn TP, Fenn KM, Nusbaum HC, Margoliash D (2008) Consolidation of sensorimotor learning during sleep. Learn Mem 15:815-819.

Cardin JA, Schmidt MF (2003) Song system auditory responses are stable and highly tuned during sedation, rapidly modulated and unselective during wakefulness, and suppressed by arousal. J Neurophysiol 90:28842899.

Chi Z, Margoliash D (2001) Temporal precision and temporal drift in brain and behavior of zebra finch song. Neuron 32:899-910.

Dave AS, Margoliash D (2000) Song replay during sleep and computational rules for sensorimotor vocal learning. Science 290:812-816.

Dave AS, Yu AC, Margoliash D (1998) Behavioral state modulation of auditory activity in a vocal motor system. Science 282:2250-2254.

Dave AS, Yu AC, Gilpin JJ, Margoliash D (1999) Methods for chronic neuronal ensemble recordings in singing birds. In: Methods for simultaneous neuronal ensemble recording (Nicolelis MAL, ed), pp 101-120. Boca Raton, FL: CRC.

Derégnaucourt S, Mitra PP, Feher O, Pytte C, Tchernichovski O (2005) How sleep affects the developmental learning of bird song. Nature 433:710-716.

Dinges DF (1990) Are you awake? Cognitive performance and reverie during the hypnopompic state. In: Sleep and cognition (Bootzin R, Kihlstrom J, Schacter D, eds), pp 159-175. Washington, DC: American Psychological Association.

Elemans CP, Mead AF, Rome LC, Goller F (2008) Superfast vocal muscles control song production in songbirds. PLoS One 3:e2581.

Fenn KM, Nusbaum HC, Margoliash D (2003) Consolidation during sleep of perceptual learning of spoken language. Nature 425:614-616.

Fischer S, Hallschmid M, Elsner AL, Born J (2002) Sleep forms memory for finger skills. Proc Natl Acad Sci U S A 18:11987-11991.

Frank MG, Issa NP, Stryker MP (2001) Sleep enhances plasticity in the developing visual cortex. Neuron 30:275-287.

Glaze CM, Troyer TW (2006) Temporal structure in zebra finch song: Implications for motor coding. J Neurosci 26:991-1005.

Hahnloser RH, Kozhevnikov AA, Fee MS (2002) An ultra-sparse code underlies the generation of neural sequences in a songbird. Nature 419:65-70.

Hahnloser RH, Kozhevnikov AA, Fee MS (2006) Sleep-related neural activity in a premotor and a basal-ganglia pathway of the songbird. J Neurophysiol 96:794-812.

Hessler NA, Doupe AJ (1999) Social context modulates singing-related neural activity in the songbird forebrain. Nat Neurosci 2:209-211.

Hoffman KL, McNaughton BL (2002) Coordinated reactivation of distributed memory traces in primate neocortex. Science 297:2070-2073.

Jewett ME, Wyatt JK, Ritz-De Cecco A, Khalsa SB, Dijk DJ, Czeisler CA (1999) Time course of sleep inertia dissipation in human performance and alertness. J Sleep Res 8:1-8.

Ji D, Wilson MA (2007) Coordinated memory replay in the visual cortex and hippocampus during sleep. Nat Neurosci 10:100-107.

Kao MH, Doupe AJ, Brainard MS (2005) Contributions of an avian basal ganglia-forebrain circuit to real-time modulation of song. Nature 433:638-643.

Karni A, Tanne D, Rubenstein BS, Askenasy JJ, Sagi D (1994) Dependence on REM sleep of overnight improvement of a perceptual skill. Science 265:679-682.

Kozhevnikov AA, Fee MS (2007) Singing-related activity of identified HVC neurons in the zebra finch. J Neurophysiol 97:4271-4283.

Leonardo A (2004) Experimental test of the birdsong error-correction model. Proc Natl Acad Sci U S A 101:16935-16940.

Leonardo A, Fee MS (2005) Ensemble coding of vocal control in birdsong. J Neurosci 25:652-661.

Leonardo A, Konishi M (1999) Decrystallization of adult birdsong by perturbation of auditory feedback. Nature 399:466-470.

Lewicki MS (1994) Bayesian modeling and classification of neural signals. Neural Comp 6:1005-1030.

Long MA, Fee MS (2008) Using temperature to analyse temporal dynamics in the songbird motor pathway. Nature 456:189-194.

Low PS, Shank SS, Sejnowski TJ, Margoliash D (2008) Mammalian-like features of sleep structure in zebra finches. Proc Natl Acad Sci U S A 105: 9081-9086.

Maquet P, Laureys S, Peigneux P, Fuchs S, Petiau C, Phillips C, Aerts J, Del 
Fiore G, Degueldre C, Meulemans T, Luxen A, Franck G, Van Der Linden M, Smith C, Cleeremans A (2000) Experience-dependent changes in cerebral activation during human REM sleep. Nat Neurosci 3:831-836.

Mooney R (1992) Synaptic basis for developmental plasticity in a birdsong nucleus. J Neurosci 12:2464-2477.

Nadasdy Z, Hirase H, Czurko A, Csicsvari J, Buzsaki G (1999) Replay and time compression of recurring spike sequences in the hippocampus. J Neurosci 19:9497-9507.

Nick TA, Konishi M (2001) Dynamic control of auditory activity during sleep: correlation between song response and EEG. Proc Natl Acad Sci U S A 98:14012-14016.

Nordeen KW, Nordeen EJ (1992) Auditory feedback is necessary for the maintenance of stereotyped song in adult zebra finches. Behav Neural Biol 57:58-66.

Olveczky BP, Andalman AS, Fee MS (2005) Vocal experimentation in the juvenile songbird requires a basal ganglia circuit. PLoS Biol 3:e153.

Peigneux P, Laureys S, Fuchs S, Collette F, Perrin F, Reggers J, Phillips C, Degueldre C, Del Fiore G, Aerts J, Luxen A, Maquet P (2004) Are spatial memories strengthened in the human hippocampus during slow wave sleep? Neuron 44:535-545.

Pennartz CM, Lee E, Verheul J, Lipa P, Barnes CA, McNaughton BL (2004) The ventral striatum in off-line processing: ensemble reactivation during sleep and modulation by hippocampal ripples. J Neurosci 24:6446-6456.

Peyrache A, Khamassi M, Benchenane K, Wiener SI, Battaglia FP (2009) Replay of rule-learning related neural patterns in the prefrontal cortex during sleep. Nat Neurosci 12:919-926.

Poe GR, Nitz DA, McNaughton BL, Barnes CA (2000) Experiencedependent phase-reversal of hippocampal neuron firing during REM sleep. Brain Res 855:176-180.

Qin YL, McNaughton BL, Skaggs WE, Barnes CA (1997) Memory reprocessing in corticocortical and hippocampocortical neuronal ensembles. Philos Trans R Soc Lond B Biol Sci 352:1525-1533.

Rauske PL, Shea SD, Margoliash D (2003) State and neuronal classdependent reconfiguration in the avian song system. J Neurophysiol 89:1688-1701.

Reis J, Schambra HM, Cohen LG, Buch ER, Fritsch B, Zarahn E, Celnik PA, Krakauer JW (2009) Noninvasive cortical stimulation enhances motor skill acquisition over multiple days through an effect on consolidation. Proc Natl Acad Sci U S A 5:1590-1595.

Roberts TF, Klein ME, Kubke MF, Wild JM, Mooney R (2008) Telencephalic neurons monosynaptically link brainstem and forebrain premotor networks necessary for song. J Neurosci 28:3479-3489.
Scott BB, Lois C (2007) Developmental origin and identity of song system neurons born during vocal learning in songbirds. J Comp Neurol 502:202-214.

Scotto-Lomassese S, Rochefort C, Nshdejan A, Scharff C (2007) HVC interneurons are not renewed in adult zebra finches. Eur J Neurosci $25: 1663-1668$.

Shank SS, Margoliash D (2009) Sleep and sensorimotor integration during early vocal learning in a songbird. Nature 458:73-77.

Simpson HB, Vicario DS (1990) Brain pathways for learned and unlearned vocalizations differ in zebra finches. J Neurosci 10:1541-1556.

Sober SJ, Brainard MS (2009) Adult birdsong is actively maintained by error correction. Nat Neurosci 12:927-931.

Sossinka R, Böhner J (1980) Song types in the zebra finch (Poephila guttata castanotis). Z Tierpsychol 53:123-132.

Spiro JE, Dalva MB, Mooney R (1999) Long-range inhibition within the zebra finch song nucleus RA can coordinate the firing of multiple projection neurons. J Neurophysiol 81:3007-3020.

Stickgold R, James L, Hobson JA (2000) Visual discrimination requires sleep after training. Nat Neurosci 3:1237-1238.

Trevisan MA, Mindlin GB, Goller F (2006) Nonlinear model predicts diverse respiratory patterns of birdsong. Phys Rev Lett 96:058103.

Turrigiano GG, Nelson SB (2004) Homeostatic plasticity in the developing nervous system. Nat Rev Neurosci 5:97-107.

Vyazovskiy VV, Cirelli C, Pfister-Genskow M, Faraguna U, Tononi G (2008) Molecular and electrophysiological evidence for net synaptic potentiation in wake and depression in sleep. Nat Neurosci 11:200-2008.

Vyazovskiy VV, Olcese U, Lazimy YM, Faraguna U, Esser SK, Williams JC, Cirelli C, Tononi G (2009) Cortical firing and sleep homeostasis. Neuron 63:865-878.

Wagner U, Gais S, Haider H, Verleger R, Born J (2004) Sleep inspires insight. Nature 427:352-355.

Walker MP, Brakefield T, Morgan A, Hobson JA, Stickgold R (2002) Practice with sleep makes perfect: sleep-dependent motor skill learning. Neuron 35:205-211.

Wilson MA, McNaughton BL (1994) Reactivation of hippocampal ensemble memories during sleep. Science 265:676-679.

Yu AC, Margoliash D (1996) Temporal hierarchical control of singing in birds. Science 273:1871-1875.

Zann RA (1985) Ontogeny of the zebra finch distance call: I. Effects of crossfostering to Bengalese finches. Z Tierpsych 68:1-23. 